\title{
DESIGN AND KINEMATIC ANALYSIS OF A RRPR ROBOT ARM
}

\author{
Yasar, S. A. \\ Manufacturing Engineering, Gazi University, \\ Ankara, Turkey,
}

\begin{abstract}
In this study, kinematic and design analysis were performed of a four degrees of freedom robot arm with Denavit-Hartenberg notation. This robot arm can be programmed for many purposes. For example, this purpose could be locating the right places of books in libraries. Finding a book may be hard because of the wrong placement by visitors. It is clear that a robotic system is a need for reducing the manpower and providing high accuracy in many applications. The difference of this robot arm is one of the joints is prismatic joint instead of revolute joint. Owing to the linear motion of the joint, positioning error will be reduced and also robot arm will be able to approach from a safer distance to prevent from accidents. Design and analysis were performed on MATLAB Simulink.
\end{abstract}

\section{Keywords}

Robotics, Kinematic Analysis, Robot Arm, Denavit-Hartenberg Notation

\section{INTRODUCTION}

Robotics is a field of modern technology that crosses traditional engineering boundaries. Understanding the complexity of robots and their applications requires knowledge of mechanical engineering, electrical engineering, systems and industrial engineering, computer science, economics, and mathematics [1]. Robots, initially meant for entertainment and fun, have dramatically changed the human life in recent years. The current technological revolution in robotics and automation has transformed the concept by accomplishing industrial tasks in a safer, optimized and much more efficient manner. Robotic manipulators are composed of rigid links interconnected by joints and are designed to function like a human arm but with enhanced strength and payload capacity [2]. Since the advent of the industrial robot, the world has been captivated by the idea of an automatically controlled agent that could make anything [3].

\section{CLASSIFICATION OF ROBOTS}

Robots can be classified according various criteria, such as degrees of freedom, kinematic structure, drive technology, workspace geometry, motion characteristics, and control [1]. The number of degrees of freedom that a manipulator possesses is the number of independent position variables that would have to be specified in order to locate all parts of the mechanism. This is a general term used for any mechanism [4]. Another classification of robots is according to their structural topologies. A robot is said to be a serial robot or serial (open-loop) manipulator if its kinematic structure takes the form of an open loop-chain, a parallel manipulator if it is made of a closed-loop chain, and hybrid manipulator if it is consists of both open and closed-loop

\author{
Korkut, I. \\ Manufacturing Engineering, Gazi University, \\ Ankara, Turkey,
}

chains [1]. The samples of serial manipulator (a) and parallel manipulator (b) are shown in Figure 1.

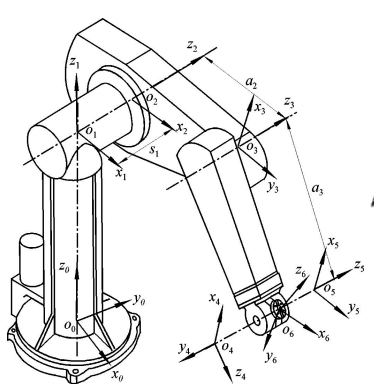

(a)

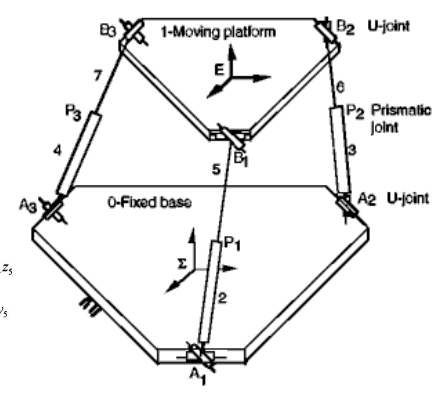

(b)
Figure 1. Serial (a) and parallel (b) manipulator samples [5,6].

Manipulators can also be classified by their drive technology. The three popular drive technologies are electric, hydraulic and pneumatic. Most manipulators use either electric dc servomotors or stepper motors, because they are clean and relatively easy to control. However, when high-speed and/or high-load-carrying capabilities are needed, hydraulic or pneumatic drive is preferred [7].

Robots come in many sizes and shapes. The type of coordinate system used by the manipulator also varies. The type of coordinate system, the arrangement of joints, and the length of the manipulator's segments all help determine the shape of the work envelope. To identify the maximum work area, a point on the robot's wrist is used, rather than the tip of the gripper or the end of the tool bit. Therefore, the work envelope is slightly larger when the tip of the tool is considered. Work envelopes vary from one manufacturer to another, depending on the exact design of the manipulator arm. Combining different configurations in a single robot can result in another set of possible work envelopes. Before choosing a particular robot configuration, the application must be studied carefully to determine the precise work envelope requirements. Some work envelopes have a geometric shape; others are irregular. One method of classifying a robot is by the configuration of its work envelope. Some robots may be equipped for more than one configuration. The four major configurations are: revolute, Cartesian, cylindrical, and spherical. Each configuration is used for specific applications [8]. 


\section{DESIGN AND KINEMATIC ANALYSIS OF A RRPR ROBOT ARM}

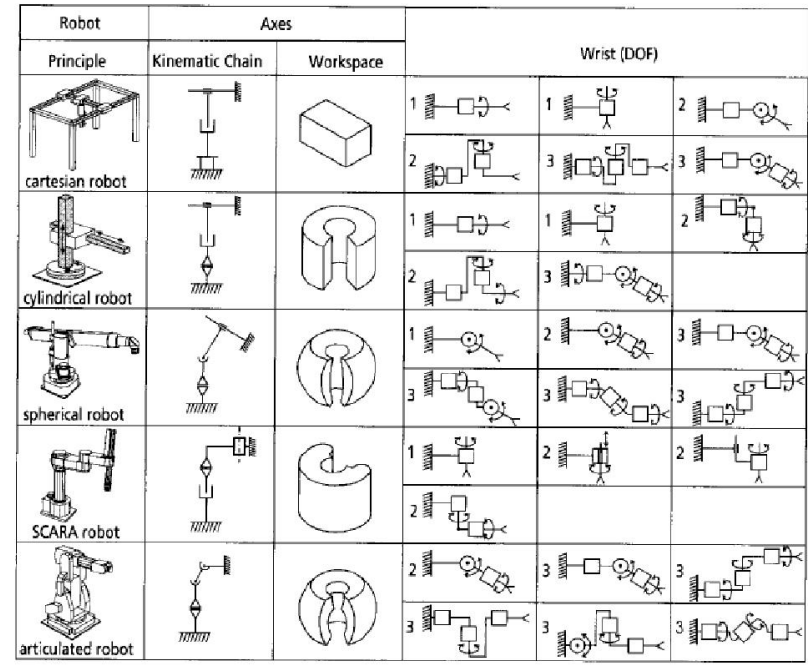

Figure 2. Robot types.

Robot manipulators can also be classified according to their nature of motion in planar, spherical and spatial [1].

Robots can also be classified by control method into servo (closed loop control) and non-servo (open loop control) robots. Servo controlled robots are further classified according to the method that the controller uses to guide the end-effector. Non-servo robots are essentially open-loop devices whose movement is limited to predetermined mechanical stops, and they are primarily used for materials transfer [9].

\section{METHOD}

In this study, a four degrees of freedom robot arm was designed and kinematic analysis was performed with Denavit-Hartenberg notation. A robot has to have four different quantities for each link. Two of them describe the link itself, and the others describe the relation to an adjacent link. Usually a joint variable and three different link parameters have to be described, either revolute joint or prismatic joint. This convention is called DenavitHartenberg notation.

\subsection{Design}

The robot arm shown in Figure 3 is designed which has a four degrees of freedom. Beside the revolute joints, it also has a prismatic joint. It was decided to use a one degree of freedom servo motor for wrist movement. The manufacturing material may differ due to the limitations such as carrying capacity.

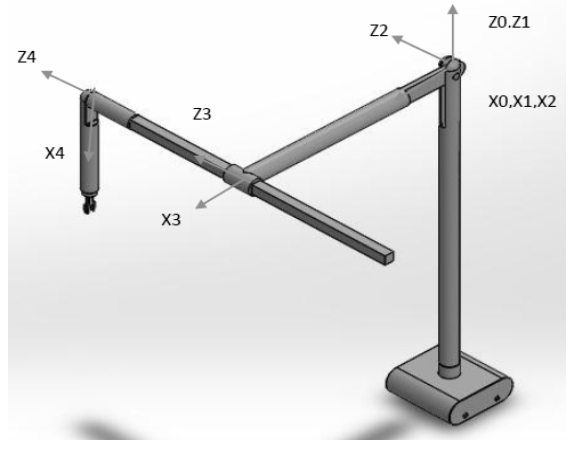

Figure 3. Robot arm design.
Kinematics is the science of motion that treats the subject without regard to the forces that cause it. Within the science of kinematics, one studies the position, the velocity, the acceleration, and all higher order derivatives of the position variables (with respect to time or any other variable(s)). Hence, the study of the kinematics of manipulators refers to all the geometrical and time-based properties of the motion [4].

\subsection{Kinematic Analysis}

The link parameters of the designed manipulator by using Denavit-Hartenberg notation is shown in Table 1. (3R1P or RRPR)

Table 1. The link parameters of the manipulator

\begin{tabular}{|c|c|c|c|c|}
\hline $\mathbf{i}$ & $\alpha_{i-1}$ & $a_{i-1}$ & $d_{i}$ & $\theta_{i}$ \\
\hline 1 & 0 & 0 & 0 & $\theta_{1}$ \\
\hline 2 & $\frac{\pi}{2}$ & 0 & 0 & $\theta_{2}$ \\
\hline 3 & 0 & $l_{2}$ & $d_{3}$ & 0 \\
\hline 4 & 0 & 0 & $l_{3}$ & $\theta_{4}$ \\
\hline
\end{tabular}

\subsubsection{Forward Kinematics}

The forward or direct kinematics is the transformation of kinematic information from the robot joint variable space to the Cartesian coordinate space. Finding the end-effector position and orientation for a given set of joint variables is the main problem in forward kinematics. This problem can be solved by determining transformation matrices ${ }^{0} T_{i}$ to describe the kinematic information of link (i) in the base link coordinate frame. The traditional way of producing forward kinematic equations for robotic manipulators and frames. Hence, the forward kinematics is basically transformation matrix manipulation [9].

By using the parameters on Table 1, the transformation matrices between $\{0\}$ and $\{4\}$ links are;

${ }_{4}^{0} T=\left[\begin{array}{cccc}r_{11} & r_{12} & r_{13} & P_{x} \\ r_{21} & r_{22} & r_{23} & P_{y} \\ r_{31} & r_{32} & r_{33} & P_{z} \\ 0 & 0 & 0 & 1\end{array}\right]={ }_{1}^{0} T_{2}^{1} T_{3}^{2} T_{4}^{3} T$

${ }_{4}^{0} T=\left[\begin{array}{cccc}c_{1} c_{24} & -c_{1} s_{24} & s_{1} & c_{1} c_{2} l_{2}+s_{1}\left(l_{3}+d_{3}\right) \\ s_{1} c_{24} & -s_{1} s_{24} & -c_{1} & s_{1} c_{2} l_{2}-c_{1}\left(l_{3}+d_{3}\right) \\ s_{24} & c_{24} & 0 & s_{2} l_{2} \\ 0 & 0 & 0 & 1\end{array}\right]$

Matrix components are;

$$
\begin{aligned}
& r_{11}=c_{1} c_{2} c_{4}-c_{1} s_{1} s_{4}=c_{1} c_{24} \\
& r_{12}=-c_{1} c_{2} s_{4}-c_{1} s_{2} c_{4}=-c_{1} s_{24} \\
& r_{13}=s_{1} \\
& r_{21}=s_{1} c_{2} c_{4}-s_{1} s_{2} s_{4}=s_{1} c_{24} \\
& r_{22}=-S_{1} c_{2} s_{4}-s_{1} s_{2} c_{4}=-S_{1} s_{24} \\
& r_{23}=-c_{1}
\end{aligned}
$$




$$
\begin{aligned}
& r_{31}=s_{2} c_{4}+c_{2} s_{4}=s_{24} \\
& r_{32}=-s_{2} s_{4}+c_{2} c_{4}=c_{24} \\
& r_{33}=0 \\
& P_{x}=c_{1} c_{2} l_{2}+s_{1} l_{3}+s_{1} d_{3} \\
& P_{y}=s_{1} c_{2} l_{2}-c_{1} l_{3}-c_{1} d_{3} \\
& P_{z}=s_{2} l_{2}
\end{aligned}
$$

\subsubsection{Inverse Kinematics}

The determination of the joint variables reduces to solving a set of nonlinear coupled algebraic equations. Although there is no standard and generally applicable method to solve the inverse kinematic problem. There are a few analytic and numerical methods to solve the problem. The main difficulty of inverse kinematic is the multiple solutions [9].

By using $(1,1)$ and $(2,1)$ components of transformation matrix (3.2);

$$
\begin{aligned}
& r_{11}=c_{1} c_{24} \\
& r_{21}=s_{1} c_{24}
\end{aligned}
$$

By using these equations $\theta_{1}$ angle is;

$$
\frac{s_{1}}{c_{1}}=\frac{\frac{r_{21}}{c_{24}}}{\frac{r_{11}}{c_{24}}}=\frac{r_{21}}{r_{11}} \Rightarrow \theta_{1}=a \tan 2\left(r_{21}, r_{11}\right)
$$

By multiplying ${ }_{4}^{0} T$ matrix with ${ }_{1}^{0} T^{-1}$ matrix;

$$
\begin{aligned}
& { }_{1}^{0} T^{-1}{ }_{4}^{0} T={ }_{1}^{0} T^{-1}{ }_{1}^{0} T_{2}^{1} T_{3}^{2} T_{4}^{3} T \\
& {\left[\begin{array}{cccc}
c_{1} & s_{1} & 0 & 0 \\
-s_{1} & c_{1} & 0 & 0 \\
0 & 0 & 1 & 0 \\
0 & 0 & 0 & 1
\end{array}\right]\left[\begin{array}{ccccc}
r_{11} & r_{12} & r_{13} & P_{x} \\
r_{21} & r_{22} & r_{23} & P_{y} \\
r_{31} & r_{32} & r_{33} & P_{z} \\
0 & 0 & 0 & 1
\end{array}\right]=\left[\begin{array}{cccc}
c_{24} & -s_{24} & 0 & c_{2} l_{2} \\
0 & 0 & -1 & -l_{3}-d_{3} \\
s_{24} & c_{24} & 0 & s_{2} l_{2} \\
0 & 0 & 0 & 1
\end{array}\right]}
\end{aligned}
$$

By using multiplied matrix components $(1,4)$ and $(2,4)$ with result matrix components $(1,4)$ and $(2,4)$;

$$
\begin{aligned}
& c_{2} l_{2}=c_{1} P_{x}+s_{1} P_{y} \\
& s_{2} l_{2}=P_{z}
\end{aligned}
$$

By using these equations $\theta_{2}$ angle is;

$$
\frac{s_{2}}{c_{2}}=\frac{\frac{P_{z}}{l_{2}}}{\frac{c_{1} P_{x}+s_{1} P_{y}}{l_{2}}}=\frac{P_{z}}{c_{1} P_{x}+s_{1} P_{y}} \Rightarrow \theta_{2}=a \tan 2\left(P_{z}, c_{1} P_{x}+s_{1} P_{y}\right)
$$

By using $(3,1)$ and $(3,2)$ components of the matrix in equation 3.2;

$$
\frac{s_{24}}{c_{24}}=\frac{r_{31}}{r_{32}} \Rightarrow \theta_{2}+\theta_{4}=a \tan 2\left(r_{31}, r_{32}\right)
$$

Using equation 3.22 too, $\theta_{4}$ angle is;

$$
\theta_{4}=a \tan 2\left(r_{31}, r_{32}\right)-a \tan 2\left(P_{z}, c_{1} P_{x}+s_{1} P_{y}\right)
$$

After $\theta_{1}, \theta_{2}$ and $\theta_{4}$ degrees are determined, by using equation 3.19 for determining $d_{3}$;

$$
\begin{aligned}
& -l_{3}-d_{3}=-s_{1} P_{x}+c_{1} P_{y} \\
& d_{3}=s_{1} P_{x}-c_{1} P_{y}-l_{3}
\end{aligned}
$$

All variables are found.

\subsubsection{Velocity Analysis}

${ }_{1}^{0} R=\left[\begin{array}{ccc}c_{1} & -s_{1} & 0 \\ s_{1} & c_{1} & 0 \\ 0 & 0 & 1\end{array}\right] \Rightarrow{ }_{0}^{1} R=\left[\begin{array}{ccc}c_{1} & s_{1} & 0 \\ -S_{1} & c_{1} & 0 \\ 0 & 0 & 1\end{array}\right]$
${ }_{2}^{1} R=\left[\begin{array}{ccc}c_{2} & -s_{2} & 0 \\ 0 & 0 & 1 \\ s_{2} & c_{2} & 0\end{array}\right] \Rightarrow{ }_{1}^{2} R=\left[\begin{array}{ccc}c_{2} & 0 & s_{2} \\ -S_{2} & 0 & c_{2} \\ 0 & 1 & 0\end{array}\right]$
${ }_{3}^{2} R=\left[\begin{array}{ccc}1 & 0 & 0 \\ 0 & 1 & 0 \\ 0 & 0 & 1\end{array}\right] \Rightarrow{ }_{2}^{3} R=\left[\begin{array}{ccc}1 & 0 & 0 \\ 0 & 1 & 0 \\ 0 & 0 & 1\end{array}\right]$
${ }_{4}^{3} R=\left[\begin{array}{ccc}c_{4} & -s_{4} & 0 \\ s_{4} & c_{4} & 0 \\ 0 & 0 & 1\end{array}\right] \Rightarrow{ }_{3}^{4} R=\left[\begin{array}{ccc}c_{4} & s_{4} & 0 \\ -s_{4} & c_{4} & 0 \\ 0 & 0 & 1\end{array}\right]$

${ }^{i+1} \omega_{i+1}={ }_{i}^{i+1} R^{i} \omega_{i}+\dot{\theta}_{i+1}{ }^{i+1} z_{i+1}$ equation is used for determining angular velocity. In addition to that, ${ }^{i+1} v_{i+1}={ }_{i}^{i+1} R\left({ }^{i} v_{i}+{ }^{i} \omega_{i} x^{i} P_{i+1}\right)$ equation is used for determining linear velocity.

$$
\begin{aligned}
& { }^{1} \omega_{1}={ }_{0}^{1} R^{0} \omega_{0}+\dot{\theta}_{1}^{1} z_{1}=\left[\begin{array}{lll}
0 & 0 & \dot{\theta}_{1}
\end{array}\right]^{T} \\
& { }^{1} v_{1}={ }_{0}^{1} R\left({ }^{0} v_{0}+{ }^{0} \omega_{0} x^{0} P_{1}\right)=\left[\begin{array}{lll}
0 & 0 & 0
\end{array}\right]^{T} \\
& { }^{2} \omega_{2}={ }_{1}^{2} R^{1} \omega_{1}+\dot{\theta}_{2}^{2} z_{2}==\left[\begin{array}{lll}
s_{2} \dot{\theta}_{1} & c_{2} \dot{\theta}_{1} & \dot{\theta}_{2}
\end{array}\right]^{T} \\
& { }^{2} v_{2}={ }_{1}^{2} R\left({ }^{1} v_{1}+{ }^{1} \omega_{1} x^{1} P_{2}\right)=\left[\begin{array}{lll}
0 & 0 & 0
\end{array}\right]^{T} \\
& { }^{3} \omega_{3}={ }_{2}^{3} R^{2} \omega_{2}=\left[\begin{array}{lll}
s_{2} \dot{\theta}_{1} & c_{2} \dot{\theta}_{1} & \dot{\theta}_{2}
\end{array}\right]^{T}
\end{aligned}
$$

${ }^{3} v_{3}={ }_{2}^{3} R\left({ }^{2} v_{2}+{ }^{2} \omega_{2} x^{2} P_{3}\right)+d_{3} z_{3}=\left[\begin{array}{c}c_{2} \dot{\theta}_{1} d_{3} \\ \dot{\theta}_{2} l_{2}-s_{2} \dot{\theta}_{1} d_{3} \\ d_{3}-c_{2} \dot{\theta}_{1} l_{2}\end{array}\right]$$$
{ }^{4} \omega_{4}={ }_{3}^{4} R^{3} \omega_{3}+\dot{\theta}_{4}^{4} z_{4}=\left[\begin{array}{llll}
s_{24} \dot{\theta}_{1} & c_{24} \dot{\theta}_{1} & \dot{\theta}_{2}+\dot{\theta}_{4}
\end{array}\right]^{T}
$$

${ }^{4} v_{4}={ }_{3}^{4} R\left({ }^{3} v_{3}+{ }^{3} \omega_{3} x^{3} P_{4}\right)+d_{4} z_{4}=\left[\begin{array}{c}c_{24} \dot{\theta}_{1}\left(l_{3}+d_{3}\right)+s_{4} \dot{\theta}_{2} l_{2} \\ c_{4} \dot{\theta}_{2} l_{2}-s_{24} \dot{\theta}_{1}\left(l_{3}+d_{3}\right) \\ d_{3}-c_{2} \dot{\theta}_{1} l_{2}+l_{3}\end{array}\right]$ 


\section{DESIGN AND KINEMATIC ANALYSIS OF A RRPR ROBOT ARM}

\section{CONCLUSIONS}

In this paper, a four degrees of freedom robot arm which has linear motion ability was designed and the kinematic analysis of the robot arm were shown by using Denavit-Hartenberg notation. Calculated equations were imported on MATLAB Simulink. It is tested and confirmed. Same results were obtained. Dynamic analysis of the robot arm can carry out in following works. Also mobility and perception options can be considered in following works instead of targeting a location.

\section{REFERENCES}

[1] Pandilov, Z., Dukovski, V. 2014. Comparison of the characteristics between serial and parallel robots. Acta Technica Corviniensis-Bulletin of Engineering 7(1), 143.

[2] Manzoor, S., Islam, R. U., Khalid, A., Samad, A., Iqbal, J. 2014. An open-source multi-DOF articulated robotic educational platform for autonomous object manipulation. Robotics and Computer-Integrated Manufacturing 30(3), 351-362.

[3] Keating, S., Oxman, N. 2013. Compound fabrication: A multi-functional robotic platform for digital design and fabrication. Robotics and Computer-Integrated Manufacturing 29(6), 439-448.

[4] Craig, J.J. 2005. Introduction to Robotics Mechanics and Control Horton, M.J., Pearson Prentice Hall, New Jersey, USA.

[5] Fang, Y., Tsai, L. W. 2003. Feasible motion solutions for serial manipulators at singular configurations. Journal of Mechanical Design 125(1), 61-69.

[6] Bonev, I. A., Zlatanov, D. 2001. The mystery of the singular SNU translational parallel robot. ParalleMIC Reviews (4).

[7] Tsai, L. W. 1999. Robot analysis: the mechanics of serial and parallel manipulators. John Wiley \& Sons.

[8] Ross, L., Fardo, S., Masterson, J., Towers, R. 2011. Robotics: Theory and Industrial Application. GoodheartWillcox.

[9] Jazar, R. N. 2010. Theory of applied robotics: kinematics, dynamics, and control. Springer Science \& Business Media. 